\title{
Analog-Digital Beamforming for Tunable-Load MIMO by Mutual Coupling Exploitation
}

\author{
Ang $\mathrm{Li}^{\dagger}$, Christos Masouros ${ }^{\dagger}$, and Mathini Sellathurai* \\ Dept. of Electronic and Electrical Eng., University College London, London, U.K. ${ }^{\dagger}$ \\ Signal Process. and Commun. Research Group, Heriot-Watt University, EH14 4AS, U.K.* \\ Email: \{ang.li.14, c.masouros\}@ucl.ac.uk, M.Sellathurai@hw.ac.uk
}

\begin{abstract}
In this paper, we propose a joint analog-digital (AD) beamforming scheme that exploits the mutual coupling effect to further improve the performance of multi-user multiple-input single-output (MU-MISO) systems. We firstly propose a joint AD scheme by iteratively optimizing the beamforming vectors in the digital domain and the load impedances of each antenna element in the analog domain. We further propose a lowcomplexity decoupled AD scheme, where the mutual coupling exploitation can be efficiently applied upon existing beamformers. The practical implementation of the proposed schemes is also discussed. Numerical results show that the proposed schemes can achieve an improved performance compared to the case with fixed mutual coupling.
\end{abstract}

Index Terms-MU-MISO, mutual coupling, analog-digital beamforming, joint optimization.

\section{INTRODUCTION}

Transmit beamforming schemes can transfer the computational burden from the user side to the transmitter side, and therefore have received extensive research attention [1]. Due to the low computational complexity, closed-form linear beamforming schemes have become popular among all the beamforming schemes [1]-[3]. On the other hand, transmit beamforming schemes based on convex optimization have drawn increasing research attention due to the performance advantages and flexibility [4][5]. One popular form of the optimization known as signal-to-interference-plus-noise ratio (SINR) balancing targets at maximizing the minimum SINR subject to a total power consumption [4]. Another form is to minimize the total power consumption subject to a minimum SINR requirement [6]. These two problems are shown to be inverse problems, based on which semidefinite programming (SDP) and iterative designs have been proposed to efficiently solve the optimization problems.

Most existing beamforming schemes are based on the assumption of an ideal antenna array, where there is no spatial correlation or mutual coupling effect among antenna elements. Nevertheless, in a practical scenario where the antenna spacing is small, the spatial correlation and mutual coupling have a significant impact on the system performance. The effect of spatial correlation has been experimentally studied in [7]-[9], and the beamforming designs for the spatially correlated channels are proposed in [10][11]. The effect of mutual coupling has been investigated in [12]-[14], where it is shown that the mutual coupling usually degrades the detection performance of MIMO systems. In order to alleviate the performance loss, compensation techniques have been proposed [15]-[18], where in most cases the compensation of mutual coupling is realized by forming a compensation matrix. Apart from these compensation techniques, in [19]-[21] the mutual coupling is shown to be capable of improving the performance of singleuser MIMO systems by exploiting the concept of constructive interference. Nevertheless, the exploitation of mutual coupling with transmit beamforming for multi-user transmission has not yet been studied.

In this paper, we propose a joint analog-digital (AD) beamforming scheme to exploit the mutual coupling effect to further improve the performance of MU-MISO systems. In the proposed scheme, each antenna element is equipped with a tunable load impedance (for example a varactor) so that the mutual coupling can be controlled. With the proposed iterative design, the beamforming vectors in the digital domain and the values of each tunable load in the analog domain are jointly optimized, leading to an improved performance. We further propose a low-complexity decoupled AD scheme, where the beamforming vectors in the digital domain are firstly obtained with existing schemes, based on which we optimize the values of each load impedance. Realistic constraints are considered for the optimization of the load impedances, and we further discuss the implementation of the proposed AD schemes in practice. It is shown in the numerical results that the proposed schemes with mutual coupling exploitation can achieve significant performance gains over conventional cases with fixed mutual coupling.

Notations: $a$, a, and A denote scalar, vector and matrix, respectively. $\mathbb{E}\{\cdot\},(\cdot)^{H}$, and $(\cdot)^{-1}$ denote expectation, conjugate transpose, and inverse of a matrix respectively. $\|\cdot\|$ denotes the Frobenius norm, and $\mathbf{I}$ is the identity matrix. We denote $\mathbf{0}$ as a zero matrix or vector. $\mathcal{C}^{n \times n}$ represents an $n \times n$ matrix in the complex set, and $\operatorname{diag}(\cdot)$ denotes the conversion of a vector into a diagonal matrix with the values on its main diagonal. $\Re(\cdot)$ and $\Im(\cdot)$ denote the real and imaginary part of a complex number, respectively.

\section{System MODEL}

\section{A. Downlink System Model}

We consider an MU-MISO downlink system, where a base station (BS) with $N_{t}$ antennas communicates with $K$ singleantenna users simultaneously, and $K \leq N_{t}$. Assuming a 
transmit beamforming matrix $\mathbf{P} \in \mathcal{C}^{N_{t} \times K}$, the received signal for the $k$-th user can be obtained as

$$
y_{k}=\mathbf{h}_{k} \mathbf{P} \mathbf{s}+n_{k},
$$

where $\mathbf{s}=\left[s_{1}, \ldots, s_{K}\right]^{T} \in \mathcal{C}^{K \times 1}$ denotes the symbol vector, and $\mathbf{h}_{k} \in \mathcal{C}^{1 \times N_{t}}$ is the channel vector for user $k . n_{k}$ represents the additive white Gaussian noise (AWGN) and each $n_{k} \sim$ $\mathcal{C N}\left(0, \sigma^{2}\right)$, where $\sigma^{2}$ is the noise power. For simplicity we have assumed $\mathbb{E}\left\{\mathbf{s s}^{H}\right\}=\mathbf{I}$.

\section{B. Channel Model with Correlation and Mutual Coupling}

When the antenna spacing is small, the spatial correlation and mutual coupling effect cannot be ignored in modelling the channel. Therefore, in this paper we assume a geometric semicorrelated Rayleigh flat-fading channel, where the correlation and mutual coupling are considered at the BS side. Following [22]-[26], the channel vector $\mathbf{h}_{k}$ is modelled as

$$
\mathbf{h}_{k}=\mathbf{g}_{k} \mathbf{A}_{k} \mathbf{Z} \text {. }
$$

In (2), $\mathbf{g}_{k} \in \mathcal{C}^{1 \times M}$ and $M$ denotes the number of directions of departure (DoDs), with each entry $\mathbf{g}_{k}(i) \sim \mathcal{C N}(0,1)$ that models the Rayleigh component of the channel. $\mathbf{A}_{k} \in \mathcal{C}^{M \times N_{t}}$ contains $M$ steering vectors at the transmit side that model the spatial correlation. For a uniform linear array (ULA), as considered in this paper, $\mathbf{A}_{k}$ is given by

$$
\mathbf{A}_{k}=\left[\mathbf{a}^{T}\left(\varphi_{k, 1}\right), \ldots, \mathbf{a}^{T}\left(\varphi_{k, M}\right)\right]^{T},
$$

and each $\mathbf{a}\left(\varphi_{k, m}\right)$ can be expressed as

$$
\mathbf{a}\left(\varphi_{k, m}\right)=\left[1, e^{j 2 \pi d \sin \varphi_{k, m}}, \ldots, e^{j 2 \pi\left(N_{t}-1\right) d \sin \varphi_{k, m}}\right] .
$$

In (4), $d$ denotes the antenna spacing normalized by the carrier wavelength, and $\varphi_{k, m}$ is the angle of departure (AoD) that follows the uniform distribution in $\left[-\varphi_{0}, \varphi_{0}\right][22]$.

In (2), the mutual coupling effect is fully characterized by a mutual coupling matrix $\mathbf{Z} \in \mathcal{C}^{N_{t} \times N_{t}}$. Based on [13][20][27], the mutual coupling matrix $\mathbf{Z}$ with tunable loads can be expressed as

$$
\mathbf{Z}\left(\mathbf{z}_{L}\right)=\left[z_{A} \cdot \mathbf{I}+\operatorname{diag}\left(\mathbf{z}_{L}\right)\right]\left[\boldsymbol{\Gamma}+\operatorname{diag}\left(\mathbf{z}_{L}\right)\right]^{-1},
$$

where $z_{A}$ is the antenna impedance, $\mathbf{z}_{L}=\left[z_{L_{1}}, \ldots, z_{L_{N_{t}}}\right]^{T} \in$ $\mathcal{C}^{N_{t} \times 1}$ denotes the tunable load vector that is to be optimized. $\boldsymbol{\Gamma}$ represents the mutual impedance matrix, and is obtained as

$$
\boldsymbol{\Gamma}=\left[\begin{array}{ccccc}
z_{A} & z_{m_{1}} & z_{m_{2}} & \cdots & z_{m_{N_{t}-1}} \\
z_{m_{1}} & z_{A} & z_{m_{1}} & \ddots & \vdots \\
z_{m_{2}} & z_{m_{1}} & \ddots & \ddots & z_{m_{2}} \\
\vdots & \ddots & \ddots & \ddots & z_{m_{1}} \\
z_{m_{N_{t}-1}} & \cdots & z_{m_{2}} & z_{m_{1}} & z_{A}
\end{array}\right]
$$

In (6), $z_{m_{k}}$ denotes the mutual impedance of two antenna elements with the distance of $k d$. In this paper, we assume a uniform dipole antenna array, and the calculation of $z_{A}$ and each $z_{m_{k}}$ can be obtained by the induced electromagnetic-field (EMF) method based on $d[13][20]$.

\section{Joint Analog-Digital Beamforming Scheme}

In this section, the proposed joint $\mathrm{AD}$ beamforming scheme is introduced, where the transmitter structure at the $\mathrm{BS}$ is shown in Fig. 1. With tunable loads on each antenna element, the mutual coupling effect can be controlled. We then jointly optimize the beamforming vectors and each load $z_{L_{i}}$ such that the resulting beamformers and load impedances are jointly optimal.

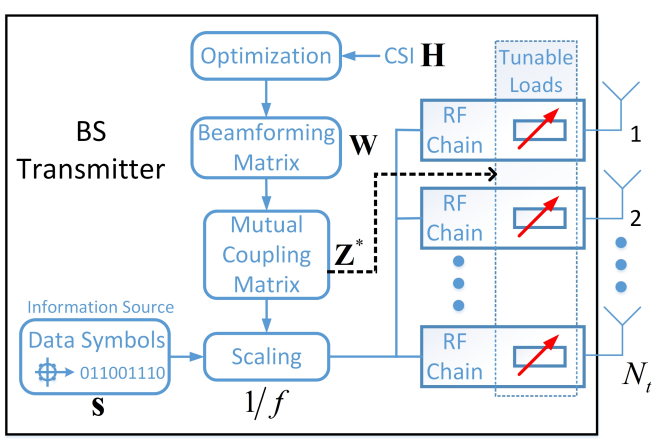

Fig. 1: Transmitter Structure

To exploit the mutual coupling, we firstly rewrite the channel vector $\mathbf{h}_{k}$ as

$$
\mathbf{h}_{k}=\mathbf{d}_{k} \mathbf{Z}
$$

where $\mathbf{d}_{k}=\mathbf{g}_{k} \mathbf{A}_{k}$. Then, (1) can be transformed into

$$
y_{k}=\mathbf{d}_{k} \mathbf{Z P s}+n_{k}
$$

based on which we construct the proposed beamformers as

$$
\mathbf{P}=\frac{1}{f} \cdot \mathbf{Z}^{-1} \mathbf{W}
$$

In (9), following the formulation of closed-form beamforming schemes $f=\left\|\mathbf{Z}^{-1} \mathbf{W}\right\|$ is the scaling factor that ensures the signal power remains unchanged after beamforming. With the proposed beamforming matrix $\mathbf{P}$, the mutual coupling effect is fully eliminated in the channel, while its effect on $\mathbf{P}$ and the system performance is fully characterized by the resulting scaling factor $f$. With $\mathbf{P}$ given by (9), the received SINR for user $k$ can be expressed as

$$
\gamma_{k}=\frac{\left|\mathbf{d}_{k} \mathbf{w}_{k}\right|^{2}}{\sum_{i \neq k}\left|\mathbf{d}_{k} \mathbf{w}_{i}\right|^{2}+f^{2} \sigma^{2}},
$$

where $\mathbf{W}=\left[\mathbf{w}_{1}, \ldots, \mathbf{w}_{K}\right]$ and $f$ can then be regarded as a noise amplification factor. It can be observed in (10) that $\gamma_{k}$ is relevant to both $f$ and each $\mathbf{w}_{k}$, and therefore we can maximize the minimum $\gamma_{k}$ by jointly optimize $\mathbf{w}_{k}$ and each load impedance $z_{L_{i}}$, on condition that each load value $z_{L_{i}}$ is practically feasible. It has been shown in [28][29] that the real part of each tunable load $z_{L_{i}}$ should be non-negative in a practical antenna array to enable the antenna radiation, based on which we can construct the optimization problem as

$$
\begin{array}{ll}
\mathcal{P}_{1}: & \max _{\mathbf{z}_{L}, \mathbf{W}} \min _{k} \gamma_{k} \\
\text { s.t. } & \Re\left(z_{L_{i}}\right) \geq 0, \forall i \in \mathcal{I}
\end{array}
$$


where $\mathcal{I}=\left\{1,2, \ldots, N_{t}\right\}$. The optimization problem $\mathcal{P}_{1}$ is not convex due to $f=\left\|\mathbf{Z}^{-1} \mathbf{W}\right\|$ in (10), and it is therefore difficult to directly solve it. Nevertheless, $\mathcal{P}_{1}$ can be transformed into a bi-convex problem, which means that it is convex with respect to $\mathbf{W}$ when $\mathbf{Z}$ is fixed and vice verse. Therefore, in the following we propose to alternately optimize $\mathbf{W}$ and each $z_{L_{i}}$.

\section{A. Solving $\mathbf{W}$ when $\mathbf{z}_{L}$ is fixed}

When each $z_{L_{i}}$ is fixed, $\mathcal{P}_{1}$ is reduced to a digital SINR balancing problem, obtained as

$$
\begin{aligned}
& \mathcal{P}_{2}: \max _{\mathbf{W}} \min _{k} \gamma_{k} \\
& \text { s.t. }\left\|\mathbf{Z}^{-1} \mathbf{W}\right\|^{2}=1
\end{aligned}
$$

In $\mathcal{P}_{2}$, for simplicity we have assumed $f=1$ as we can always scale $\mathbf{W}$ to satisfy this, while the optimization on $f$ is performed by optimizing $\mathbf{z}_{L}$ in the analog domain. To solve $\mathcal{P}_{2}$, we firstly consider the following SINR balancing problem, which is expressed as

$$
\begin{array}{ll}
\mathcal{P}_{3}: & \max _{\mathbf{W}} \min _{k} \gamma_{k} \\
\text { s.t. } & \left\|\mathbf{Z}^{-1} \mathbf{W}\right\|^{2} \leq 1
\end{array}
$$

where we optimize $\mathbf{W}$ while $\mathbf{Z}$ is fixed. Based on the SINR expression in (10) and [4][5], it is easily observed that the power constraint on $\mathcal{P}_{3}$ is strictly active when the optimality is achieved, which means that $\mathcal{P}_{3}$ is indeed equivalent to $\mathcal{P}_{2}$. Therefore, in the following we propose to solve $\mathcal{P}_{3}$ instead. Compared to conventional SINR balancing problem, the only difference in $\mathcal{P}_{3}$ is that the power constraint is on $\mathbf{Z}^{-1} \mathbf{W}$ instead of $\mathbf{W}$. Thanks to the fact that $\mathbf{Z}^{-1}$ is considered as fixed, this problem can be efficiently solved by existing schemes for solving conventional SINR balancing problems [4][6], and therefore the details are omitted for brevity.

\section{B. Solving $\mathbf{z}_{L}$ when $\mathbf{W}$ is fixed}

It is observed from (10) that $f$ can be regarded as a noise amplification factor. Therefore, when $\mathbf{W}$ is fixed, based on (10) $\mathcal{P}_{1}$ is equivalent to optimizing each $z_{L_{i}}$ such that the resulting $f$ is minimized, which leads to the following optimization problem

$$
\begin{array}{ll}
\mathcal{P}_{4}: & \min _{\mathbf{z}_{L}}\left\|\mathbf{Z}^{-1} \mathbf{W}\right\|^{2} \\
\text { s.t. } & \Re\left(z_{L_{i}}\right) \geq 0, \forall i \in \mathcal{I}
\end{array}
$$

To solve $\mathcal{P}_{4}$, based on (5) we firstly obtain $\mathbf{Z}^{-1}$ as

$$
\begin{aligned}
\mathbf{Z}^{-1} & =\left[\boldsymbol{\Gamma}+\operatorname{diag}\left(\mathbf{z}_{L}\right)\right]\left[z_{A} \cdot \mathbf{I}+\operatorname{diag}\left(\mathbf{z}_{L}\right)\right]^{-1} \\
& =\left[\boldsymbol{\Gamma}+\operatorname{diag}\left(\mathbf{z}_{L}\right)\right] \operatorname{diag}(\mathbf{z})
\end{aligned}
$$

where

$$
\mathbf{z}=\left[\frac{1}{z_{1}}, \ldots, \frac{1}{z_{N_{t}}}\right]^{T}
$$

and each $z_{i}=z_{A}+z_{L_{i}}$. We further denote $\Theta=$ $\operatorname{diag}\left(\left[\theta_{1}, \ldots, \theta_{N_{t}}\right]^{T}\right)$ with each $\theta_{i}=\frac{z_{m_{1}}}{z_{i}}$, and $\mathbf{Z}^{-1}$ can be decomposed into

$$
\mathbf{Z}^{-1}=\mathbf{B \Theta}+\mathbf{I}
$$

where $\mathbf{B}$ is given by

$$
\mathbf{B}=\left[\begin{array}{ccccc}
0 & 1 & \frac{z_{m_{2}}}{z_{m_{1}}} & \cdots & \frac{z_{m_{N_{t}-1}}}{z_{m_{1}}} \\
1 & 0 & 1 & \ddots & \vdots \\
\frac{z_{m_{2}}}{z_{m_{1}}} & 1 & \ddots & \ddots & \frac{z_{m_{2}}}{z_{m_{1}}} \\
\vdots & \ddots & \ddots & \ddots & 1 \\
\frac{z_{m_{N_{t}-1}}}{z_{m_{1}}} & \cdots & \frac{z_{m_{2}}}{z_{m_{1}}} & 1 & 0
\end{array}\right] .
$$

With $\theta_{i}=\frac{z_{m_{1}}}{z_{i}}$, we can express each tunable load as

$$
z_{L_{i}}=\frac{z_{m_{1}}}{\theta_{i}}-z_{A}
$$

and the constraint $\Re\left(z_{L_{i}}\right) \geq 0$ can be transformed into

$$
\begin{aligned}
& \Re\left(\frac{z_{m_{1}}}{\theta_{i}}\right) \geq \Re\left(z_{A}\right) \\
\Rightarrow & \Re\left(\theta_{i}\right) \Re\left(z_{m_{1}}\right)+\Im\left(\theta_{i}\right) \Im\left(z_{m_{1}}\right) \geq\left\|\theta_{i}\right\|^{2} \Re\left(z_{A}\right) .
\end{aligned}
$$

With (17) and (20), the optimization problem $\mathcal{P}_{4}$ can be finally expressed in a convex form as

$$
\begin{aligned}
& \mathcal{P}_{5}: \min _{\Theta}\|\mathbf{B \Theta} \mathbf{W}+\mathbf{W}\|^{2} \\
& \text { s.t. } \Re\left(\theta_{i}\right) \Re\left(z_{m_{1}}\right)+\Im\left(\theta_{i}\right) \Im\left(z_{m_{1}}\right) \geq\left\|\theta_{i}\right\|^{2} \Re\left(z_{A}\right), \forall i \in \mathcal{I}
\end{aligned}
$$

$\mathcal{P}_{5}$ is a least-squares problem and can be efficiently solved via convex optimization tools. The optimal loads can be obtained by (19) and the resulting optimal mutual coupling matrix is obtained as

$$
\mathbf{Z}^{*}=\left(\mathbf{B} \Theta^{*}+\mathbf{I}\right)^{-1} \text {. }
$$

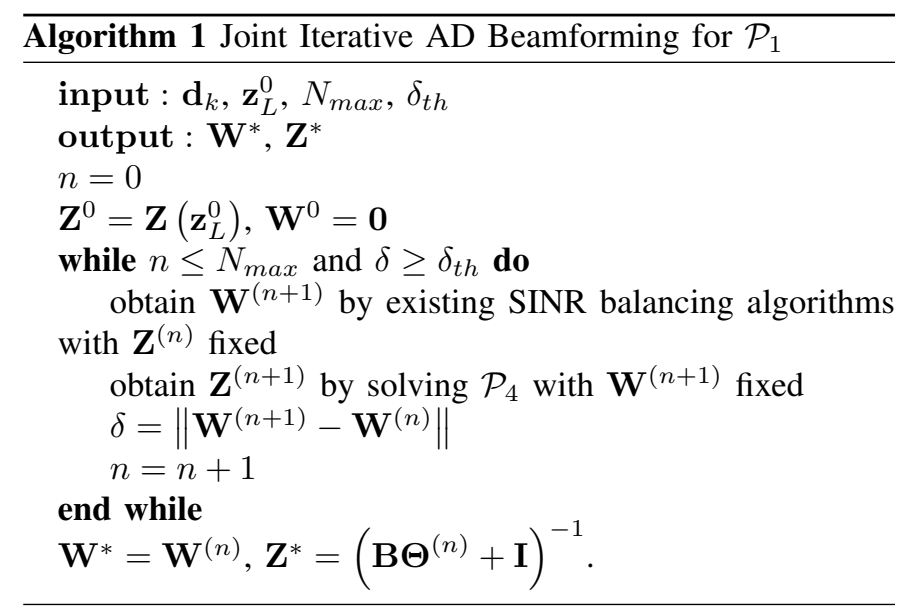

\section{Joint Iterative Algorithm}

Based on the above, the optimality can be achieved by iteratively optimizing $\mathbf{W}$ and $\mathbf{z}_{L}$ until convergence or a maximum number of iterations is reached. We then summarize the above iterative design in Algorithm 1, where we denote $\mathbf{z}_{L}^{0}$ as the initial load vector and $N_{\max }$ as the maximum iteration number. $\delta$ denotes the convergence accuracy and $\delta_{t h}$ is the accuracy threshold. 
Convergence: In Algorithm 1, firstly the two sub-problems have the same objective function in $\mathcal{P}_{1}$, then we have observed that the obtained $\mathbf{W}^{(n)}$ by $\mathcal{P}_{3}$ and $\mathbf{Z}^{(n)}$ by $\mathcal{P}_{5}$ are optimally solved within each iteration. Therefore, Algorithm 1 is guaranteed to converge [30][31], and we have $f^{*}=1$ when the optimality is reached.

\section{Decoupled Analog-Digital Beamforming SCHEME}

Due to the iterative design, the proposed joint $\mathrm{AD}$ scheme is of high computational complexity. To alleviate the computational burden in practice, in this section we propose a low-complexity decoupled AD scheme, where the optimization of the digital beamformers is firstly conducted, followed by the optimization of the load vector $\mathbf{z}_{L}$. Furthermore, with the decoupled approach, closed-form beamformers such as ZF can be combined with the mutual coupling exploitation, which greatly reduces the complexity. To be specific, for the decoupled approach we still construct the beamforming matrix as in (9), and we denote $\mathbf{W}_{d}$ as the digital beamformers. We firstly obtain $\mathbf{W}_{d}$, based on which we minimize $f$ by optimizing each $z_{L_{i}}$. The decoupled SINR balancing beamforming scheme is then summarized in Algorithm 2 below.

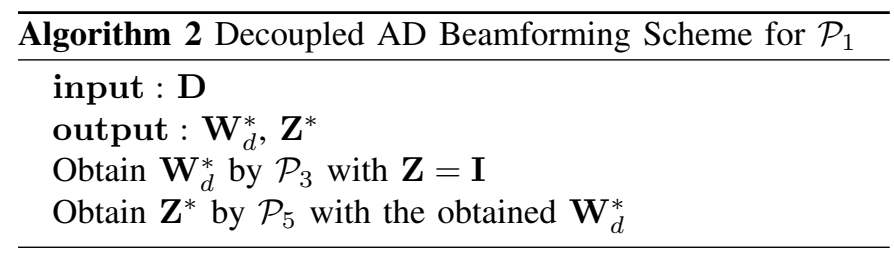

For the case of closed-form beamforming schemes such as $\mathrm{ZF}, \mathbf{W}_{d}^{*}$ is directly substituted with the closed-form expressions. Then, the optimization of the load impedances follows a similar step to $\mathcal{P}_{4}$ and $\mathcal{P}_{5}$ of Section III. B and can be efficiently solved.

\section{Practical Implementation Aspects}

It is easy to observe from Section III and IV that similar to conventional beamforming schemes, the proposed $\mathrm{AD}$ schemes require the knowledge of each $\mathbf{d}_{k}$ to perform the optimization. We further note that in the presence of mutual coupling, we can only obtain $\mathbf{h}_{k}$ with channel estimation techniques at the BS. To extract $\mathbf{d}_{k}$ from $\mathbf{h}_{k}$, we can firstly set each load impedance to a reference value (for example $\left.z_{L_{i}}^{0}=50 \Omega, \forall i \in \mathcal{I}\right)$ and perform channel estimation, where we denote the reference load vector and mutual coupling matrix as $\mathbf{z}_{L}^{0}$ and $\mathbf{Z}_{0}$, respectively. Then, with $\boldsymbol{\Gamma}$ typically known at the BS, $\mathbf{Z}_{0}$ can be obtained and based on (7) we can then obtain $\mathbf{d}_{k}=\mathbf{h}_{k} \mathbf{Z}_{0}^{-1}$, and the proposed schemes can be applied.

On the other hand, the proposed scheme requires the adaptation of each tunable load dependent on the variations of the channels. It has been shown in [32] that advanced semiconductor technologies such as ferroelectric-based varactors can support a very fast tuning speed. An adaptive matching network can then be employed with an automated impedance tuning unit with ferroelectric-based varactors to facilitate the proposed scheme. Moreover, the application of adaptive impedance tuning has already been employed in the electronically steerable parasitic antenna radiators (ESPARs) and has been verified by experiments [33].

\section{NUMERICAL RESULTS}

We present the numerical results based on the Monte Carlo simulations in this section. For the channel modelling, the number of DoDs is $M=50, \phi_{0}=\pi / 8$ and the antenna spacing is assumed $d=0.25$ (which is equivalent to $\lambda / 4$ ). The initial value of each tunable load is $z_{L_{i}}^{0}=50 \Omega, \forall i \in \mathcal{I}$. We compare SINR balancing beamforming with closed-form ZF beamforming, and for clarity we denote 'SB AD Joint', 'SB AD Decoupled' and 'SB with MC' as the SINR balancing beamforming with the proposed joint $\mathrm{AD}$ scheme, decoupled AD scheme, and conventional fixed mutual coupling. For ZF, only the decoupled approach can be applied.

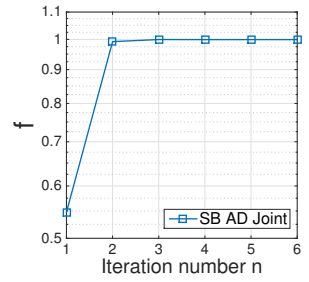

(a) Value of $f$

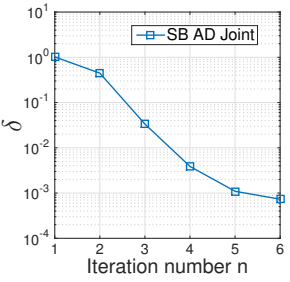

(b) Value of $\delta$
Fig. 2: Convergence of the joint AD scheme, $N_{t}=K=4$, $N_{\max }=6, \delta_{t h}=10^{-2}$

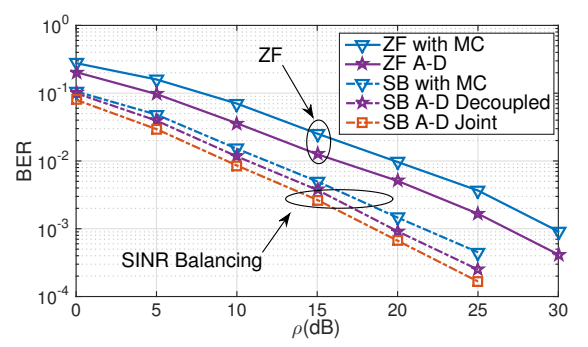

Fig. 3: BER comparison, $N_{t}=K=4, N_{\max }=6, \delta_{t h}=$ $10^{-2}$, QPSK

In Fig. 2 the convergence of the joint AD scheme is shown, where we plot the values of both $f$ and $\delta$ with respect to the iteration number $n$. It can be observed that the proposed $\mathrm{AD}$ scheme is convergent within $n=4$ iterations, and $f^{*}=1$ when the convergence is reached.

In Fig. 3 we compare the bit error rate (BER) performance of the proposed $\mathrm{AD}$ scheme, where QPSK modulation is applied. It can be observed that for both ZF and SINR balancing, the proposed $\mathrm{AD}$ scheme can achieve an improved performance over the conventional case with fixed mutual coupling. The joint $\mathrm{AD}$ scheme is shown to achieve the best BER performance.

The sum rate for each beamforming scheme is compared in Fig. 4, where the sum rate is obtained based on the received SINR in (10). It is shown that both the joint and decoupled scheme offer a higher rate performance compared to conventional digital beamforming. 


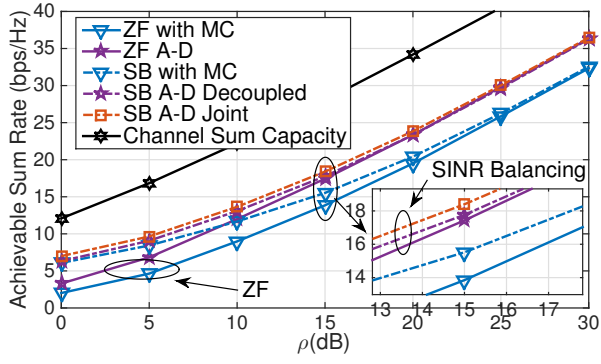

Fig. 4: Sum rate, $N_{t}=K=4, N_{\max }=6, \delta_{t h}=10^{-2}$

\section{CONCLUSION}

In this paper, we investigate the mutual coupling for MUMISO, where we show that the mutual coupling effect can be beneficial by judiciously selecting the load values of each antenna element. A joint AD beamforming scheme and a lowcomplexity decoupled AD scheme are then proposed to exploit the mutual coupling. Numerical results show that compared to conventional cases where the mutual coupling effect is detrimental, with the proposed schemes the mutual coupling can improve the system performance.

\section{ACKNOWLEDGMENT}

This work was supported by the Royal Academy of Engineering, U.K., the Engineering and Physical Sciences Research Council (EPSRC) project EP/M014150/1, and the China Scholarship Council (CSC).

\section{REFERENCES}

[1] C. Windpassinger, R. Fischer, T. Vencel, and J. Huber, "Precoding in Multiantenna and Multiuser Communications," IEEE Trans. Wireless Commun., vol. 3, no. 4, pp. 1305-1316, July 2004.

[2] C. B. Peel, B. M. Hochwald, and A. L. Swindlehurst, "A VectorPerturbation Technique for Near-Capacity Multiantenna Multiuser Communication-Part I: Channel Inversion and Regularization," IEEE Trans. Commun., vol. 53, no. 1, pp. 195-202, Jan. 2005.

[3] C. Masouros, "Correlation Rotation Linear Precoding for MIMO Broadcast Communications," IEEE Trans. Sig. Process., vol. 59, no. 1, pp. 252-262, Jan. 2011.

[4] A. Wiesel, Y. C. Eldar, and S. Shamai (Shitz), "Linear Precoding via Conic Optimization for Fixed MIMO Receivers," IEEE Trans. Sig. Process., vol. 54, no. 1, pp. 161-176, Jan. 2006.

[5] M. Schubert and H. Boche, "Solution of the Multiuser Downlink Beamforming Problem with Individual SINR Constraints," IEEE Trans. Veh. Tech., vol. 53, no. 1, pp. 18-28, Jan. 2004.

[6] F. Rashid-Farrokhi, K. Liu, and L. Tassiulas, "Transmit Beamforming and Power Control for Cellular Wireless Systems," IEEE J. Sel. Areas Commun., vol. 16, no. 8, pp. 1437-1450, Oct. 1998.

[7] M. T. Ivrlac, W. Utschick, and J. A. Nossek, "Fading Correlations in Wireless MIMO Communication Systems," IEEE J. Sel. Areas Commun., vol. 21, no. 5, pp. 819-828, June 2003.

[8] A. M. Tulino, A. Lozano, and S. Verdu, "Impact of Antenna Correlation on the Capacity of Multiantenna Channels," IEEE Trans. Inf. Theory, vol. 51, no. 7, pp. 2491-2509, July 2005.

[9] H. Liu, Y. Song, and R. C. Qiu, "The Impact of Fading Correlation on the Error Performance of MIMO Systems over Rayleigh Fading Channels," IEEE Trans. Wireless Commun., vol. 4, no. 5, pp. 2014-2019, Sept. 2005.

[10] H. R. Bahrami and T. Le-Ngoc, "Precoder Design based on the Channel Correlation Matrices," IEEE Trans. Wireless Commun., vol. 5, no. 12, pp. 3579-3587, Dec. 2006.
[11] J. Akhtar and D. Gesbert, "Spatial Multiplexing over Correlated MIMO Channels with a Closed-form Precoder," IEEE Trans. Wireless Commun., vol. 4, no. 5, pp. 2400-2409, Sept. 2005.

[12] I. Gupta and A. Ksienski, "Effect of Mutual Couplong on the Performance of Adaptive Arrays," IEEE Trans. Ant. Propag., vol. 31, no. 5, pp. 785-791, Sept. 1983.

[13] C. A. Balanis, Antenna Theory: Analysis and Design, 3rd ed. WileyBlackwell, May 2005.

[14] A. A. Aouda and S. G. Haggman, "Effect of Mutual Coupling on Capacity of MIMO Wireless Channels in High SNR," Progress in Electro-magnetics Research, vol. 65, pp. 27-40, 2005.

[15] Z. Li, Z. Du, M. Takahashi, K. Saito, and K. Ito, "Reducing Mutual Coupling of MIMO Antennas with Parasitic Elements for Mobile Terminals," IEEE Trans. Ant. Propag., vol. 60, no. 2, pp. 473-481, Feb. 2012.

[16] H. Aliakbarian, S. Farsi, D. Schreurs, B. Nauwelaers, and G. A. E. Vandenbosch, "Mutual Coupling Reduction between Planar Antennas by Using a Simple Microstrip U-Section," IEEE Ant. Wireless Propag. Lett., vol. 11, pp. 1501-1503, Dec. 2012.

[17] G. Moreno, H. M. Bernety, and A. B. Yakovlev, "Reduction of Mutual Coupling between Strip Dipole Antennas at Terahertz Frequencies with an Elliptically Shaped Graphene Monolayer," IEEE Ant. Wireless Propag. Lett., vol. 15, pp. 1533-1536, Dec. 2015.

[18] J. Rubio, J. F. Izquierdo, and J. Corcoles, "Mutual Coupling Compensation Matrices for Transmitting and Receiving Arrays," IEEE Trans. Ant. Propag., vol. 63, no. 2, pp. 839-843, Feb. 2015.

[19] A. Li and C. Masouros, "Mutual Coupling Exploitation for Point-topoint MIMO by Constructive Interference," in 2017 IEEE International Conference on Communications (ICC), Paris, France, accepted.

[20] — , "Exploiting Constructive Mutual Coupling in P2P MIMO by Analog-Digital Phase Alignment," IEEE Trans. Wireless Commun., DOI: 10.1109/TWC.2017.2657631, to appear, 2017.

[21] A. Li, C. Masouros, and C. B. Papadias, "MIMO Transmission for Single-fed ESPAR with Quantized Loads," IEEE Trans. Commun., DOI: 10.1109/TCOMM.2017.26922242017.

[22] C. Masouros, M. Sellathurai, and T. Ratnarajah, "Large-Scale MIMO Transmitters in Fixed Physical Spaces: The Effect of Transmit Correlation and Mutual Coupling," IEEE Trans. Commun., vol. 61, no. 7, pp. 2794-2804, July 2013.

[23] C. Wang and R. D. Murch, "Adaptive Downlink Multi-user MIMO Wireless Systems for Correlated Channels with Imperfect CSI," IEEE Trans. Wireless Commun., vol. 5, no. 9, pp. 2435-2446, Sept. 2006.

[24] S. Biswas, C. Masouros, and T. Ratnarajah, "Performance Analysis of Large Multi-User MIMO Systems with Space-Constrained 2D Antenna Arrays," IEEE Trans. Wireless Commun., vol. 15, no. 5, pp. 3492-3505, May 2016.

[25] A. Garcia-Rodriguez and C. Masouros, "Exploiting the Increasing Correlation of Space Constrained Massive MIMO for CSI Relaxation," IEEE Trans. Commun., vol. 64, no. 4, pp. 1572-1587, Apr. 2016.

[26] C. Masouros and M. Matthaiou, "Space-Constrained Massive MIMO: Hitting the Wall of Favourable Propagation," IEEE Commun. Lett., vol. 19, no. 5, pp. 771-774, May 2015.

[27] B. Clerckx, C. Craeye, D. V-Janvier, and C. Oestges, "Impact of Antenna Coupling on 2 x 2 MIMO Communications," IEEE Trans. Veh. Tech., vol. 56, no. 3, pp. 1009-1018, May 2007.

[28] J. Choma and W. K. Chen, Feedback Networks: Theory and Circuit Applications. USA World Scientific Publishing, May 2007.

[29] H. A. Haus, Electromagnetic Noise and Quantum Optical Measurements. Springer, Nov. 2000.

[30] M. Razaviyayn, M. Hong, and Z.-Q. Luo, "A Unified Convergence Analysis of Block Successive Minimization Methods for Nonsmooth Optimization," SIAM J. Optim, vol. 23, no. 2, pp. 1126-1153, 2013.

[31] Q. Zhang, C. He, and L. Jiang, "Per-Stream MSE based Linear Transceiver Design for MIMO Interference Channels with CSI Error," IEEE Trans. Commun., vol. 63, no. 5, pp. 1676-1689, May 2015.

[32] J.-S. Fu, "Adaptive Impedance Matching Circuits based on Ferroelectric and Semiconductor Varactors," Ph.D. dissertation, University of Michigan, 2009.

[33] O. N. Alrabadi, C. Divarathne, P. Tragas, A. Kalis, N. Marchetti, C. B. Papadias, and R. Prasad, "Spatial Multiplexing with a Single Radio: Proof-of-Concept Experiments in an Indoor Environment with a $2.6 \mathrm{GHz}$ Prototype," IEEE Commun. Lett., vol. 15, no. 2, pp. 178-180, Feb. 2011. 